Fecha de recepción: marzo 2021

Fecha de aprobación: abril 2021

Fecha publicación: mayo 2021

\section{Contribuciones inalienables: algunas reflexiones sobre las condiciones de posibilidad para la reevaluación de la evidencia etnográfica}

Gabriel D. Noel ${ }^{(1)}$

\begin{abstract}
Resumen: Los argumentos que Annette Weiner despliega en Inalienable Possessions, su principal trabajo teórico, se apoyan sobre un reexamen de la evidencia etnográfica de ciertos trabajos, sitios y perspectivas clásicas de la antropología a la luz de su propia experiencia de campo y de la movilización de conceptos y categorías analíticas contemporáneas. Más allá de los debates más conocidos en torno de algunos "reestudios" famosos de la historia de la disciplina, las (re)evaluaciones sistemáticas de argumentos etnográficos como los acometidos por Weiner siguen resultando más la excepción que la regla en la práctica de nuestro métier, muchas veces propensa reemplazar el reexamen de la evidencia con el presentismo trivial, los paralogismos ingenuos y las chicanas facciosas. Sobre esta base, el presente texto se propone utilizar el libro de Weiner para reflexionar en torno de las condiciones de posibilidad para una (re)evaluación rigurosa de la evidencia etnográfica, asi como también las de la producción de textos etnográficos que ofrezcan la posibilidad de ser reevaluados a posteriori.
\end{abstract}

Palabras clave: Annete Weiner - Teoría Antropológica - Etnografía - Reestudios.

[Resúmenes en inglés y portugués en las páginas 35-36]

(1) Gabriel D. Noel es Lic. en Antropología (UNLP), Doctor en Ciencias Sociales (UNGS), Investigador CONICET en IDAES-UNSAM y Profesor Titular en la Licenciatura en Antropología Social y Cultural de la Universidad Nacional de San Martín. Investiga sobre problemas teóricos y metodológicos del trabajo antropológico y sociológico en la pequeña y mediana escala urbana. Correo: gdnoel@gmail.com

Clifford Geertz señalaba, hace casi medio siglo, que "la antropología (...) es una ciencia cuyo progreso se caracteriza menos por un perfeccionamiento del consenso que por el refinamiento del debate" (Geertz, 1990, p. 39). Aun cuando a la luz de la desprolija realidad de las comunidades antropológicas que conocemos esta vocación progresiva pueda 
parecer un tanto optimista, tampoco puede dudarse de que en cierto sentido constituye una buena descripción del quehacer de nuestra disciplina en sus mejores momentos, esto es, en los más inspirados, rigurosos y reflexivos. Creemos, en este sentido y en relación con el tema del presente dossier, que apenas puede dudarse de que Inalienable Possessions, un texto en el cual su autora, Annette Weiner, vuelve, se detiene, reexamina y reformula ciertos trabajos, sitios y perspectivas clásicas de la antropología a partir de categorías teóricas renovadas constituye un ejemplo cabal de estos "mejores momentos" en los cuales la producción de conocimiento antropológico a partir de un debate a la vez refinado y riguroso parece darle la razón a ese desideratum geertziano. A partir de esa convicción-cuyos fundamentos y razones iremos desplegando a lo largo del presente texto- consideramos que Inalienable Possessions puede pensarse como un exemplum -en el sentido kuhniano recientemente rehabilitado por Latour (2008) - para la práctica de la antropología, es decir como una instancia particularmente lograda (y en ese mismo sentido modélica) que nos permite ilustrar algunas de las notas definitorias del ejercicio de la escritura y la argumentación en nuestro métier así como de algunas de sus operaciones fundamentales, como la presentación de los datos, la reevaluación de la evidencia y la (re)construcción de un argumento en diálogo con las tradiciones disciplinarias que nos precedieron y en relación con las cuales nos definimos. Al mismo tiempo querríamos utilizar este examen, que no podrá ser más que somero, para poner de relieve por vía de contraste ciertas limitaciones implícitas en algunas prácticas contemporáneas de la escritura y la discusión antropológica que creemos conspiran en contra del establecimiento de esa comunidad de debate utópica- irrealizable y deseable a la vez, como toda utopía que se precie de tal -de la que hablaba Geertz y sobre la base de la cual podríamos permitirnos aspirar a ese mínimo horizonte de rigor intersubjetivo -esto es de desacuerdo explícito y razonado- que nos permita sentirnos parte de una disciplina científica llamada "antropología".

Comencemos pues por una estilizada e imprescindible reconstrucción del texto y de su argumento $^{1}$. Lo que se propone Weiner en Inalienable Possessions es una vuelta sobre uno de los debates centrales de la historia de la teoría antropológica -la discusión en torno del don y el intercambio y de su lugar en la constitución del lazo social 'inaugurada’2 por Mauss y Malinowski en sus dos textos célebres y contemporáneos, el Ensayo sobre el Don y Los Argonautas del Pacífico Occidental (Malinowski, 1986; Mauss, 2009) y prolongada primero por Crimen y Costumbre en la Sociedad Salvaje (Malinowski, 1991) y más tarde por intervenciones, entre otras, de Claude Lévi-Strauss (1977), Marshall Sahlins (1972) y Pierre Clastres $(2005)^{3}$ - para mostrar, a partir de una operación metodológica inspirada en la deconstrucción derridiana ${ }^{4}$, de qué manera y hasta qué punto las controversias en torno de lo que efectivamente se intercambia encubren un suplemento oculto y más fundamental: la distinción constitutiva (y putativamente universal) entre las cosas que circulan o que pueden intercambiarse y las que no: las "posesiones inalienables" que son invocadas en el título del texto. La elisión de estas últimas en las conceptualizaciones antropológicas sobre el lugar del intercambio en el lazo social asumen para Weiner consecuencias problemáticas, puesto que son ellas y sólo ellas -o más bien la capacidad de producirlas, mantenerlas y retenerlas- las que proveen de "autenticación cosmológica" [cosmological authentication], esto es, de fundamento y legitimación ontológica a la perpetuación de la desigualdad y a la reproducción de ésta a través del tiempo. Al mismo tiempo, a la hora de explicar cómo 
es posible que una distinción tan antigua y tan central en los sistemas jurídicos tanto antiguos como contemporáneos pudo pasársele por alto a investigadores tan sutiles $-\mathrm{y}$ a la vez conocedores del derecho, sus instituciones y su historia- en el marco de una distinción específicamente jurídica, la autora echa mano de la teoría crítica feminista para revelar de qué manera y hasta qué punto la suposición irreflexiva de que es en la esfera pública, la del intercambio (que es, apenas hace falta decirlo, una esfera prominentemente masculina) donde se juegan las cosas que "verdaderamente importan" consiguió sustraerles con éxito la clave de que es con frecuencia en la correlativa esfera doméstica (y por tanto eminentemente "femenina") donde estas posesiones se producen, conservan y retienen en el marco del lugar central de las mujeres en los fenómenos ligados a la reproducción. A la luz de esta relación complementaria -o mejor aún suplementaria- las desigualdades se reproducen en el tiempo bajo la apariencia superficial de un intercambio generalizado de equivalentes y en el marco de una tensión aporética que Weiner llama la paradoja del "retener-mientras-se-da" [keeping-while-giving], es decir, de hacer circular ciertas cosas que satisfagan la demanda permanente y potencialmente irrecusable de "dar" identificada por Mauss, al tiempo que procuran sustraer otras de la circulación (las que "verdaderamente importan") que se intentan mantener en el dominio de "lo propio" a cualquier precio -en la medida en que, como hemos mencionado, de ellas depende la perpetuación de la legitimidad cosmológica de la preeminencia de quienes las detentan. Al ignorar, por tanto, esta tensión constitutiva entre lo que circula y lo que se retiene y más allá de sus protestas anti-etnocéntricas en contrario, la teoría antropológica clásica -o al menos varios de los más prominentes de entre sus practicantes del siglo pasado- habría contribuido a perpetuar y prolongar una economía política "fantasmática", heredera de Smith y de su propuesta de colocar en el intercambio la clave maestra y el fundamento último del lazo social. Sobre esta clave de lectura y a partir de las categorías teóricas ya mencionadas, Weiner habrá de realizar a lo largo del libro mencionado un magistral tour de force en el que volverá -como adelantáramos- sobre varios textos, sitios y debates clásicos de la disciplina para reexaminar y reinterpretar los datos inscriptos en ellos y reconstruir de manera a la vez provocativa e iluminadora las explicaciones (pre)existentes tanto a partir de la mirada provista por esta nueva perspectiva -lo que podríamos llamar en aras de la brevedad una "critica externa"- como por un examen de sus ambigüedades, tensiones y lagunas -la correlativa "crítica interna".

A partir de estas constataciones una primera pregunta que podríamos hacernos - no por obvia menos necesaria- sería: ¿cómo es que Weiner arriba tanto a la necesidad como a la posibilidad de esta reconstrucción? ¿Cómo y por qué ésta se vuelve necesaria por un lado y posible por el otro? La respuesta se encuentra en primer lugar, claro está, en su propia experiencia etnográfica en un área-Oceanía en general, y las Islas Trobriand en particular (Weiner, 1976) - que constituyera uno (sino el principal) de los puntos de origen del moderno trabajo de campo en nuestra disciplina, y por tanto de la identidad colectiva sobre él fundada (Stocking, 1983; Kuklick, 2011). En este sentido, su trabajo en áreas frecuentadas por la investigación antropológica precedente le permite prestar particular atención a elementos, rasgos o procesos que encuentra como resultado de su propio trabajo y que aparecen insuficiente o insatisfactoriamente explicados en los reportes de sus predecesores $^{5}$. Pero ciertamente esto no es todo: al tiempo que abre nuevos frentes e interrogantes 
sobre la base de su propio trabajo de campo, puede realizar un reexamen de los datos contenidos en las etnografías que constituyen sus antecedentes porque sus autores presentaron y documentaron en ellas una cantidad (sobre)abundante de material que aunque no necesariamente fuera pasible de interpretación e incorporación en sus formulaciones teóricas, fue de todos modos incluido como parte de un reporte que buscaba presentar una reconstrucción tan exhaustiva como fuera posible (o al menos practicable) de la "realidad observada" -ese "registro consultable" de lo dicho y de lo hecho por el ser humano al que también hiciera referencia Geertz en el texto ya citado (Geertz, 1990, p. 39). Insistimos, y aún conscientes del riesgo del pleonasmo: si Weiner puede reinterpretar y volver sobre los fenómenos presentados por sus predecesores ello es posible solamente y en la medida en que aquéllos han sido adecuadamente documentados por éstos, tanto en el caso de los detalles que resultaban pertinentes para argumentar, articular o fundamentar un punto de teoría como en el de los que no, e incluso en el de los que representaban una fuente de perplejidad, inconsistencia o potencial contradicción respecto de los restantes hechos observados y registrados ${ }^{6}$. Cuando, por el contrario, esta posibilidad de volver sobre los datos ajenos para someterlos a examen y eventualmente reinterpretarlos resulta bloqueada por una descripción insuficientemente atenta a la relación entre datos y teoría (que mantenga a la vista y permita restituir el vínculo que el autor establece entre unos y otros) y de ésta con detalles adicionales aparentemente irrelevantes o en todo caso difíciles de asimilar o relacionar con ella, resultará imposible a fortiori adjudicar la pregunta-como efectivamente ocurrió en casos como el de la controversia en torno de Tepoztlán ya citada-acerca de si las diferencias presentadas no podrían explicarse por el desfasaje temporal entre el reporte original y su sucesor más reciente. Sólo cuando las inconsistencias, los "residuos" teóricamente indigeribles o los eventos en principio irrelevantes e innecesarios -que pueblan todas esas largas y fatigosas descripciones con las cuales los lectores de otras disciplinas y los estudiantes nóveles se impacientan y se sienten tentados a saltear por irrelevantes- son incluidos en el reporte etnográfico es que se vuelven susceptibles de ser movilizados ulteriormente en discusiones, replanteos, apropiaciones o relecturas posteriores a la luz de categorías analíticas que no estaban disponibles para quien los inscribió en primer término y que pueden revelarse imprevistamente iluminadoras ${ }^{7}$, permitiendo a investigadoras competentes como Anette Weiner ponerlos a dialogar con su propia experiencia de trabajo de campo y a partir de allí volver sobre ellos y sobre los datos que presentan una mirada retrospectiva y rigurosa a los fines de producir ese "refinamiento del debate" que Geertz señalaba como motor del "progreso" antropológico.

Sin embargo, una descripción cuidadosa, atenta y exhaustiva no basta por sí sola para permitir (y mucho menos para alentar) la construcción de síntesis teóricas que aspiren a trascender la "generalización dentro del caso" -para seguir fatigando el texto de Geertz-y que nos permitan por tanto escapar a las autoimpuestas limitaciones de una antropología entendida como etnografía, y de ésta como "descripción densa" que, como veremos enseguida, aparecen como demasiado estrechas. Necesitamos, por el contrario, como bien ha señalado Fernando Balbi, reconocer y reivindicar el lugar de la comparación como recurso analítico de derecho propio en la construcción de argumentos antropológicos y con ella recuperar la aspiración de nuestra disciplina a un modicum de generalización teórica (Balbi, 2017). Coincidentemente, creemos con él que la putativa renuncia a esa vocación 
comparativa en el marco de los procesos de construcción de teoría en nuestra disciplina, a la sombra de la sucesión postgeertziana $-\mathrm{y}$ de su virtual entronización como modelo hegemónico para la práctica de la antropología- amenazó -y sigue amenazando- con encerrarla en un descriptivismo estéril que, bajo la coartada de la "descripción densa", sustrae a la etnografía de su lugar como estrategia de construcción de conocimiento científico y la vuelve, en el extremo, una suerte de forma glorificada de la crónica-inhibiendo, paradójicamente, la posibilidad de inscribirla en formulaciones lo suficientemente abstractas como para ser incorporadas o movilizadas en el marco de un "refinamiento del debate". Inalienable Possessions, en este sentido, con su lograda combinación de detalle etnográfico y vocación comparativa constituye un sugestivo contraejemplo, que muestra cabalmente el modo en que la reinterpretación de los materiales sobre el Kula que Weiner originalmente construyera a partir de sus propios datos de las Trobriand se prolonga y refuerza a partir de un movimiento de generalización e iluminación recíproca cuyo potencial heurístico y abductivo consigue persuadirnos de que lo que se está discutiendo no sólo arroja luz sobre una forma particular de la existencia humana -lo cual no es tarea menor ni que deba desmerecerse, por supuesto, como bien sabemos quienes practicamos la etnografía- sino que también predica de la constitución general de la vida social y colectiva, esto es, de una genuina "ciencia social".

\section{II}

Ahora bien: si reflexionamos con más atención sobre estas dos condiciones generales que acabamos de presentar -esto es, la adecuada presentación del material etnográfico y el recurso a la comparación como estrategia analítica por derecho propio-y que constituyen como hemos argumentado los requisitos mínimos de posibilidad de un trabajo de reconsideración, reevaluación y reconstrucción crítica como el acometido por Weiner, comenzamos a vislumbrar las putativas razones que se encontrarían detrás de la excepcionalidad relativa de una obra como Inalienable Possessions y de la singularidad que un texto de este alcance reviste en el marco de la producción antropológica contemporánea ${ }^{9}$. Como ya lo hemos anticipado -siguiendo una vez más a Balbi en el texto ya citado $(2017)^{10}$, la progresiva consolidación hegemónica de una versión de la práctica disciplinaria -la de la antropología cultural estadounidense- que procura y hasta cierto punto consigue redefinir su agenda teórica y metodológica sobre la base de una ecuación que disuelve la antropología en la etnografía y a ésta en el particularismo radical de la "descripción densa", ha tenido como consecuencia la elisión progresiva de cualquier ambición comparativa o pretensión generalizadora, las que son o bien sencillamente ignoradas o bien ironizadas con condescendencia como propias de épocas pretéritas y menos sofisticadas. Paradójicamente, sin embargo, el énfasis cuasi-exclusivo en la estrategia etnográfica como marca de identidad y autenticidad disciplinaria no implica de hecho, como pudiera suponerse, que la reconstrucción cuidadosa alentada por Malinowski, reformulada por Gluckman y la Escuela de Manchester y radicalizada por Geertz y sus epígonos encuentre regularmente las condiciones de desplegarse de manera cabal. Más bien por el contrario, muchos de los 
practicantes actuales de la disciplina en sede académica -y quisiera en aras del rigor dejar asentado antes de proseguir que hablo desde la experiencia de un investigador argentino publicando la mayor parte del tiempo en revistas latinoamericanas de ciencias socialesnos encontramos permanentemente enfrentados a un conjunto de condiciones objetivas que aunque nunca lo impiden del todo ofrecen al menos una tenaz y persistente resistencia a muchos de los hábitos y recursos que consideramos definitorios de nuestra práctica profesional como antropólogos y como etnógrafos.

Aún a riesgo de constatar lo obvio: una adecuada presentación del material de campo, que permita hacer justicia al proceso inductivo de construcción de conocimiento y a la adecuada fundamentación del vínculo que lleva de los datos empíricos a las conclusiones teóricas y viceversa, así como a sus pliegues, ambigüedades, residuos y potenciales contradicciones o perplejidades es algo que requiere espacio. Mucho espacio. Consecuentemente, los ejemplos más logrados de esta estrategia -a cuyo producto y vehículo material la historia acumulada de la disciplina ha denominado "monografía etnográfica"- encuentran en general su forma cabal o bien en el libro, o bien en papers con una extensión que según los criterios actuales -de los que pronto nos ocuparemos- aparece como inverosímil. Sólo para tomar dos ejemplos emblemáticos pertenecientes a la tradición mancuniense a la que hemos hecho repetida referencia: Analysis of a Social Situation in Modern Zululand (Gluckman, 1958) cuenta con 77 páginas (equivalentes a unas 38.000 palabras) y Kalela Dance (Mitchell, 1956) con 52 páginas (esto es, unas 26.000) y esto en la prosa sobria y económica posibilitada por la rígida sintaxis de la lengua inglesa. A la luz de estos guarismos no me cabe ninguna duda de que quienes estén familiarizados con las máximas extensiones permitidas habitualmente por las revistas de ciencias sociales de la región estarán sonriendo al momento de leer estas líneas. Volveremos en breve sobre esto.

Tales necesidades de despliegue descriptivo y argumentativo no serían de suyo particularmente problemáticas si no fuera por el hecho a la vez simultáneo y conocido de que nuestros sistemas de promoción y evaluación -e insisto, estoy hablando de la experiencia particular de los investigadores académicos de la Argentina contemporánea, aunque soy consciente de que no se aplican sólo a ella- están enfocados de manera predominante (para decirlo con cierta modestia y concesión a las formas) en la producción y publicación de papers en revistas académicas. Nada impide, por supuesto, escribir libros $-\mathrm{y}$ de hecho la casi totalidad de los investigadores lo seguimos haciendo- pero esto solo es posible a costa de un esfuerzo personal que asume la forma reflexiva de una indulgencia, un dispendio o incluso un desvío ${ }^{11}$, en la medida en que los mismos no suelen ser debidamente ponderados, no sólo en relación con el tiempo, el trabajo y la dificultad requeridas para producirlos, sino con el impacto sumamente desfavorable que les cabe en relación con la publicación de papers en revistas debidamente calificadas ${ }^{12}$. Nada de esto, por supuesto, es siquiera mínimamente novedoso como tampoco resulta original argumentar que estos criterios y modalidades de evaluación recogen estilos provenientes de otras disciplinas más prestigiosas y con mayor peso en el ecosistema científico y académico -ie. las ciencias experimentales.

¿Qué sucede por su parte con los papers? Como hemos ya insinuado en parte el mayor obstáculo que quienes practicamos la etnografía encontramos en relación con la posibilidad de publicar resultados de manera mínimamente adecuada tiene que ver con los límites 
tendencialmente decrecientes que las revistas académicas imponen a la extensión máxima de las presentaciones. Aun conociendo de sobra los riesgos de la evidencia anecdótica $-\mathrm{y}$ la facilidad con que los propios lectores pueden hacer el ejercicio me exime, calculo, de la obligación de presentar una caracterización sistemática- no creo ser el único que ha visto en la última década desmoronarse los límites superiores admitidos por los journals de quince mil a doce mil palabras, de doce mil a diez mil, y de ahí a ocho mil -sin que esto sea garantía, dicho sea de paso, de que este proceso digno de Zenón se haya detenido ${ }^{13}$. Así las cosas, la posibilidad de desplegar un argumento etnográfico more mancuniensis, esto es con la debida presentación de la evidencia, la explicitación de los pasos que llevan de ella a la reconstrucción analítica del investigador y esa inclusión supernumeraria de "residuos" no incorporados en la propuesta teórica que posibilitan el reexamen posterior y sucesivo resulta poco más que una quimera. A la hora de reducir nuestros ricos, complejos y matizados borradores iniciales a los estrechos límites de Procusto previstos por los Consejos Editoriales ${ }^{14}$ lo primero que se sacrifica, por regla general, es la riqueza y la sutileza del detalle etnográfico que queda reducido -y esto en el mejor de los casos- a un carácter meramente ilustrativo que ejemplifica una propuesta teórica que aparece en gran medida como arbitraria, y que resultará por tanto evaluada más en términos de su consistencia interna y de lo persuasivo de su retórica que de su relación con una evidencia empírica que les ha sido sustraida a sus evaluadores y, a fortiori, a sus lectores ${ }^{15}$.

La cuestión de la presentación de la evidencia suele complicarse aún más en el caso de que un investigador aspire a publicar por fuera del círculo relativamente restringido de revistas explícitamente identificadas como antropológicas o etnográficas, puesto que en el mundo más amplio de las "ciencias sociales" de las que éstas forman parte, el estilo de presentación propio de la escritura etnográfica -en la que como ha señalado Guber (2001) producto y proceso se imbrican en una relación íntima y necesaria- no siempre es conocido o reconocido, generando con frecuencia una disonancia en la cual aquello que sus practicantes contamos entre sus principales virtudes -su carácter exploratorio e inductivo, la implicación recíproca e indisoluble entre datos y teoría, la objetivación reflexiva de las dimensiones relacionales de la práctica de la investigación- son leídas como otros tantos defectos -incompetencia, falta de claridad analítica o expositiva, desconocimiento de las normas o el lenguaje del género académico y un largo etc. En consecuencia no es desusado que los referatos en estos ámbitos tiendan a transformarse en auténticas batallas campales en las que los etnógrafos nos vemos obligados una y otra vez a explicar lo que para nosotros es obvio - lo cual no suele favorecernos en la medida en que corremos el riesgo de sonar o bien pedantes o bien condescendientes o bien ambas cosas a un tiempo- que incluso cuando se resuelven en nuestro favor nos dejan con la amarga sospecha de que la victoria tuvo más que ver con razones ligadas a la Realpolitik académica que a la calidad de nuestros argumentos. Se nos dirá -y con justeza- que se encuentra disponible la alternativa ciertamente menos problemática de cerrar el círculo sobre las revistas explícitamente ligadas a la disciplina. Quizás. Pero más allá de que esa opción aparece a nuestros ojos como una forma de renuncia resignada que empobrece en cierta medida el "refinamiento del debate" al interior del campo más amplio del que formamos parte, por otro lado hemos venido constatando -y una vez más, nuestra afirmación debe relativizarse a la luz de una conjetura fundada en evidencia meramente personal, indicial y anecdóti- 
ca- una convergencia creciente en las publicaciones de la región en torno de una serie de constricciones provenientes del mundo del mainstream anglosajón, que acompañan con frecuencia a protocolos e indizaciones aunque no sean parte estricta de ellos y que entronizan como parámetros de la escritura académica estándar una serie de atributos de tipo Cargo Cult y que incluyen, junto con el minimalismo ya señalado respecto de la extensión, una estructura convencional que remeda la de las ciencias experimentales - "objetivos", "antecedentes", "materiales y métodos", "resultados" "discusión"- y una prosa rígida y desabrida que busca imitar la rígida sintaxis sujeto-verbo-objeto directo-punto-y-aparte de la lengua inglesa.

Como resultado de estas constricciones a las que nos vemos crecientemente enfrentados, nuestra posibilidad de contribuir activa o pasivamente a un debate colectivo se ven crecientemente menguadas. La desvalorización relativa y persistente del libro frente al paper por parte de las instituciones de financiación, evaluación y acreditación de la investigación científica en nuestro ámbito y el aumento correlativo de las constricciones respecto de qué cuenta como escritura académica razonable o incluso legítima en ciencias sociales nos colocan en una posición en la que la satisfacción de nuestra doble condición de posibilidad de un auténtico "refinamiento del debate" resulta cada vez más difícil de alcanzar. En consecuencia, y ante la imposibilidad efectiva de debatir en sentido pleno de la palabra -es decir a partir del examen cuidadoso de la evidencia y de su relación con las conclusiones teóricas que se alcanzan sobre su base, ya sea a partir de evidencia adicional, de una discrepancia interna del texto o de una propuesta alternativa que permita incluir en la explicación datos que la propuesta original dejara afuera- los intercambios habituales entre miembros de la comunidad antropológica se ordenan en un continuo que va de la cita (de aquellos con quienes acordamos) a la ignorancia cortés (de aquellos que no se ocupan de las "mismas" cosas que nosotros) y de allí (moviéndonos hacia el extremo negativo) a parodias que intentan pasar por crítica pero que encubren (casi siempre torpemente) lecturas presentistas que reprochan a los autores del pasado, con una indignación moral digna de mejor causa no haber movilizado nuestras categorías favoritas del presente (Stocking 1968), acompañadas casi siempre de chicanas facciosas contra "hombres de paja" a los que se les imputa afirmaciones que nadie seriamente sostuvo nunca. Por lo que al debate respecta, en tanto, todo ocurre como si hubiésemos perdido la capacidad o el interés de reconocerlo o de ponerlo en práctica y como si la dificultad creciente para satisfacer las condiciones efectivas necesarias para movilizarlo no mereciera más que una resignación callada y un acatamiento silencioso. Mutatis mutandis lo mismo puede decirse de la perspectiva de que nuestros datos y nuestros hallazgos sean (re)inscriptos en el marco de un horizonte comparativo de mayor alcance que la "generalización al interior del caso" que constituye la condición mínima (pero ciertamente no la máxima) del reporte etnográfico. Creo que fue Darcy Ribeiro quien dijo que si bien todas nuestras contribuciones teóricas estaban condenadas a quedar obsoletas o a volverse irrelevantes en el mediano a largo plazo -y a veces incluso en el corto- nuestras contribuciones descriptivas subsistirían por siempre, al menos en el sentido ya señalado al comienzo del presente texto según el cual siempre pueden volver a ser interrogadas y reinscriptas en un nuevo proyecto teórico, de manera tal de que devengan, a todos los efectos, contribuciones inalienables de y para nuestra disciplina ${ }^{16}$. 
Creemos por todo esto que Inalienable Possessions en tanto exemplum de producción y debate antropológico puede leerse como un mudo reproche o, si se prefiere, como una interrogación a la vez aguda y pertinente acerca de los modos contemporáneos de producción, circulación y publicación en nuestra disciplina. Aún a riesgo de insistir en una posición sospechosa de nostalgia -y por tanto susceptible de caer en un conservadurismo reaccionario- creemos que las pasiones que el libro de Weiner ha despertado entre tantos de sus lectores tiene que ver con una cierta condición "inactual", en el sentido nietzscheano del término. Mas "inactual” con una torsión paradójica: su condición de tal no surge del hecho de que se haya adelantado a su época sino, por el contrario, del de que en muchos sentidos luzca a nuestros ojos como un texto a la vez admirable en tanto anacrónico. Un texto que nos permite poner de relieve, por vía ostensiva y de contraste, varias de las principales limitaciones objetivas y subjetivas con las que debemos lidiar muchas veces en la práctica cotidiana y contemporánea de nuestra disciplina quienes nos identificamos con una tradición en la que la etnografía, como proceso, como género y como producto (Guber, 2001) y la comparación con vías a una generalización que nos permita realizar contribuciones a la ciencia del ser humano en sociedad y, por tanto, a ese "refinamiento del debate" con el que comenzáramos esta reflexión, forman parte indisoluble. Cierto: las condiciones objetivas materializadas en instituciones, dispositivos, criterios de evaluación, publicación y financiación existen. Pero no es menos cierto que esas instituciones, dispositivos y criterios nos tienen como actores, a veces incluso en posiciones más o menos centrales. Sin querer devenir apóstoles de voluntarismos inverosímiles o de agencias heroicas y descontextualizadas, no querríamos tampoco renunciar en nombre del cinismo a la posibilidad de que visibilizar nuestra incomodidad respecto de la imposibilidad efectiva de producir o alentar contribuciones inalienables en nuestra disciplina bien pueda ser el comienzo de una serie de prácticas destinadas (con perdón de la palabra) a restaurarlas.

\section{Notas}

1. Me apresuro a señalar que a los fines de la presente exposición simplificaré hasta la mutilación toda la discusión de Weiner sobre la reproducción, vinculada a su vez a la discusión antropológica sobre el tabú del incesto (y en particular del incesto entre siblings) que constituye el fundamento de su discusión con Lévi-Strauss y el problema del “intercambio de mujeres" que despliega en el Capítulo III de la obra citada.

2. Las scare quotes tienen que ver con el debido reconocimiento al argumento de Lygia Sigaud (2007) acerca de las ambigüedades relativas a la reconstrucción retrospectiva de Mauss y del Ensayo... como parte de una putativa "teoría del intercambio" que no necesariamente estaba presente en la formulación, circulación y recepción original del texto.

3. La discusión ha proseguido con igual intensidad luego de la intervención de Weiner, sumándose a la misma Marilyn Strathern (1990), Pierre Bourdieu (1996) y Maurice Godelier (1998), para citar solamente un puñado de las intervenciones más prominentes. 
4. Aunque significativamente $-\mathrm{y}$ en un gesto que sin duda hubiese agradado al propio Derrida- éste no sea citado en el texto más que en una referencia bibliográfica menor y por interpósita persona.

5. La obra de Weiner puede leerse en este sentido como una instancia particularmente lograda de "reestudio" -aunque como veremos en breve hay más en ella que eso. Los reestudios y las controversias resultantes-como en el caso emblemático de la primera de ellas, suscitada en torno de Tepoztlán (Redfield, 2012; Lewis, 1968, 1986) -constituyen instancias particularmente representativas del ideal geertziano ya mencionado.

6. La cuestión de qué constituye una documentación "adecuada" de la situación etnográfica está por supuesto sujeta a debate, siempre sobre el fondo común del carácter "dispendioso" y "antieconómico" de la estrategia etnográfica, en la cual la relación entre el material recogido y el explícitamente publicado es, incluso en el mejor de los casos, desmesuradamente asimétrica. Aunque por razones de brevedad no podemos entrar en detalles aquí, la cuestión de cómo y qué documentar en relación con el trabajo de campo etnográfico ha sido ampliamente discutida por Gluckman y por la denominada "Escuela de Manchester" (Evens y Handelman, 2006) en un intento por zanjar la discusión de la generación precedente $-y$ más concretamente la que tuviera lugar entre Radcliffe-Brown y Malinowski en relación con el nivel de "exhaustividad" y "detalle" requeridos por la descripción etnográfica como parte de un proceso de construcción de teoría antropológica. A modo de respuesta, el grupo del Rhodes-Livingstone Institute y del departamento de Sociología y Antropología de la Universidad de Manchester congregados en torno de Gluckman y materializado en sus Seminarios desarrollarán el que se conoce como "método del caso extendido" (Van Velsen, 1967). También puede consultarse al respecto de esta cuestión el trabajo de Quirós (2014).

7. Así -y para justificar aunque sea mediante un ejemplo la inclusión de este trabajo en una discusión más general que trata sobre los objetos y su relación con las prácticas de los actores sociales- aun cuando ya para la época en que Malinowski presentaba los resultados de la primera etapa de su trabajo de campo en Mailu el interés por los objetos y lo que hoy llamaríamos "cultura material" se encontraba en franco declinio (Stocking, 1995), éste no obstante incluyó la suficiente información como para permitir a autoras posteriores como Weiner o como muchas de nuestras contemporáneas reintroducir sus materiales en una discusión que originalmente no estaba prevista.

8. En este sentido y en contraste con lo ya señalado acerca de la deriva "particularista" de la antropología cultural estadounidense y sus réplicas epigonales, trabajos como los de Weiner representan a nuestro juicio una alternativa "durkheimiana" en la cual investigación etnográfica y generalización comparativa -como una vez más, argumentara Balbi (2017) - forman parte de una y la misma empresa de construcción de conocimiento y de teoría social.

9. Queda claro que esta singularidad no debe ser exagerada, ni nuestra hipérbole ser tomada demasiado en serio. Más allá de la hegemonía creciente del modelo estadounidense al que hemos estado haciendo referencia, la vocación comparativa no ha desaparecido nunca del todo en la producción antropológica contemporánea. Aún a riesgo de la injusticia, un texto de fines de siglo que moviliza esta vocación comparativa y que hasta cierto 
punto representa un esfuerzo análogo al de Weiner es Prey into Hunter de Maurice Bloch (1992). No cabe duda de que nuestros lectores podrán reponer sus propios exempla.

10. Véase también Fernández de Rota (2009).

11. Acompañado con frecuencia por una nada desdeñable dosis de futilidad y de culpa, en virtud de la contradicción entre la convicción surgida de una acción racional con arreglo a valores que nos impulsa a mantenernos fieles a los dispositivos de presentación y circulación propios de nuestra disciplina y la surgida de una acción racional con arreglo a fines fundada en la certeza de que al menos en teoría podríamos prescindir de ellos y mantenernos -o incluso prosperar- en el sistema a fuerza exclusiva de papers "bien colocados" 12. Asimismo, la decisión de publicar un libro no se encuentra exenta de desafíos: más allá del estrecho, desbordado y desfinanciado circuito de las editoriales académicas -que aún así realizan esfuerzos dignos de elogio y con resultados nada desdeñables-los investigadores se enfrentan al dilema de tener que financiar la publicación con sus propios recursos (ya sea para sustituir o para complementar los de proyectos escasos y drásticamente devaluados) o bien optar por concentrarse en temas y proyectos cuyo interés presumiblemente desborde el del estrecho círculo de los especialistas para alcanzar el de la opinión pública ilustrada que constituye el target de las editoriales comerciales de ciencias sociales y humanidades.

13. Reducción tanto más inexplicable cuando la mayor parte de las revistas contemporáneas han dejado de circular en papel y por lo tanto vuelven irrelevante cualquier discusión relativa a los costos de papel e impresión.

14. Siempre queda la posibilidad de intentar vulnerarlos, claro -aunque más no sea dentro de ciertos márgenes más o menos razonables- mediante una suerte de prepotencia más o menos disimulada del tipo "tómelo o déjelo", pero ésta resulta en principio accesible solamente a quienes tienen suficiente capital académico (o social o político) como para tener alguna posibilidad de salirse con la suya.

15. Asimismo, cuando se lo piensa con más detenimiento, se verifica una suerte de doble vínculo implícito en una formación disciplinar que presenta varias monografías canónicas como exempla a ser imitados y como parámetro de calidad en la producción de conocimiento (Marcus y Fischer, 1986) para luego colocar a sus practicantes en condiciones objetivas que vuelven muy poco verosímil la posibilidad de reproducirlas más allá del momento inaugural e irrepetible de la disertación doctoral.

16. A modo de consuelo, siempre quedan nuestras notas, claro, para quienes tengan la inclinación, el tiempo y la paciencia de hurgar en ellas. Pero el estatuto de publicidad de las notas es bastante más complicado que el de los trabajos publicados, no sólo por cuestiones ligadas a la posibilidad efectiva de acceder a ellas -que implica o bien una relación de confianza con el autor o bien esperar a qué el mismo muera y que las mismas sean depositadas en algún archivo de acceso más o menos público- sino también por razones ligadas a la confidencialidad. 


\section{Bibliografía}

Balbi, F. (2017). Comparación, etnografía y generalización en Anuário Antropológico, 42(1): 9-35.

Bloch, M. (1992). Prey into Hunter. The Politics of Religious Experience. Cambridge: CUP.

Bourdieu, P. (1996). Marginalia. Algumas notas adicionais sobre o dom en Mana. Estudos de Antropologia Social 2 (2): 7-20.

Clastres, P. (2005). Antropología de la violencia: la guerra en las sociedades primitivas. Buenos Aires: FCE.

Evens, T. M. S. and Handelman, D. (Eds.). (2006). The Manchester School. Practice and Ethnographic Praxis in Anthropology. NY: Berghahn Books.

Fernández de Rota y Monter, J. (2009). Una etnografía de los antropólogos en EEUU. Consecuencias de los debates posmodernos. Madrid: Akal.

Geertz, C. (1990) [1973]. Descripción Densa: hacia una teoría interpretativa de la cultura en La Interpretación de las Culturas. Barcelona: Gedisa.

Gluckman, M. (1958). Analysis of a Social Situation in Modern Zululand. NY: Humanities Press, Inc.

Godelier, M. (1998). El Enigma del Don. Buenos Aires: Paidós.

Guber, R. (2001). La Etnografía. Método, Campo y Reflexividad. Buenos Aires: Norma.

Kuklick, H. (2011). Personal Equations. Reflections on the History of Fieldwork, with Special Reference to Sociocultural Anthropology, en Isis, 102:1-33.

Latour, B. (2008). Reensamblar lo Social: Una Introducción a la Teoría del Actor-Red. Buenos Aires: Manantial.

Lévi-Strauss, C. (1977) [1950]. Introducción a la obra de Marcel Mauss, en Sociología y Antropología. Madrid: Taurus.

Lewis, O. (1968) [1960]. Tepoztlán. Un Pueblo de México. México: Moritz.

Lewis, O. (1986) [1982]. Observaciones adicionales acerca del continuo folk-urbano y la urbanización, con referencia especial a la ciudad de México en Ensayos Antropológicos. México: Grijalbo.

Malinowski, B. (1986) [1922]. Los Argonautas del Pacífico Occidental. Barcelona: Planeta - Agostini.

Malinowski, B. (1991) [1926]. Crimen y Costumbre en la Sociedad Salvaje. Barcelona: Ariel.

Marcus, G. E. y Fischer, M. (1986). La Antropología como Crítica Cultural: un Momento Experimental en las Ciencias Humanas. Buenos Aires, Amorrortu.

Mauss, M. (2009) [1923]. Ensayo sobre el Don. Forma y Función del Intercambio en las Sociedades Arcaicas. Buenos Aires: Katz.

Mitchell, J. (1956). A dança Kalela: aspectos das relaçoes sociais entre africanos urbanizados na Rodésia do Norte en Feldman-Bianco, B. (Org.) (2010) Antropologia das Sociedades Contemporâneas. Métodos. Sao Paulo: FEU-UNESP.

Quirós, J. (2014). Etnografiar Mundos Vívidos. Desafíos de Trabajo de Campo, Escritura y Enseñanza en Antropología, en Publicar, XVII: 47-65.

Redfield, R. (2012) [1930]. Tepoztlan. A Mexican Village. Seattle: Ulan Press.

Sahlins, M. (1972). On the Sociology of Primitive Exchange, en Stone Age Economics (1974). London: Routledge. 
Sigaud, L. (2007). Doxa e Crença entre os Antropólogos en Novos Estudos CEBRAP 77: $129-152$.

Stocking, G. (1968). On the Limits of 'Presentism' and 'Historicism' in the Historiography of the Behavioral Sciences, en Race, Culture and Evolution. Essays in the History of Anthropology. NY: The Free Press.

Stocking, G. (1995). After Tylor. British Social Anthropology 1888-1951. Madison: University of Wisconsin Press.

Stocking, G. (1983). The Ethnographer's Magic: Fieldwork in British Anthropology from Tylor to Malinowski, en (1992) The Ethnographer's Magic Other Essays in the History of Anthropology. Madison: University of Winsconsin Press.

Strathern, M. (1990). The Gender of the Gift. Oakland: University of California Press.

Van Velsen, J. (1967) [2010]. A Análise Situacional e o Método de Estudo de Caso Detalhado, en Feldman-Bianco, B. (Org.) (2010). Antropologia das Sociedades Contemporâneas. Métodos. Sao Paulo: FEU-UNESP.

Weiner, A. (1976). Women of Value, Men of Renown: New Perspectives in Trobriand Exchange. Austin: University of Texas Press.

Weiner, A. (1992). Inalienable Possessions. Berkeley: University of California Press.

\begin{abstract}
The arguments deployed by Annette Weiner in Inalienable Possessions, her most important theoretical achievement, are based on the reevaluation of ethnographic evidence of certain classical anthropological sites, works and perspectives from the standpoint of her own fieldwork and the mobilization of contemporary concepts and analytical categories. Beyond well-known debates about certain famous "restudies" from the history of our discipline, systematic (re)evaluation of ethnographic arguments akin to that undertaken by Weiner are still more the exception than the rule in the practice of our craft, usually too eager to replace reexamination of the evidence with trivial presentisms, naïve paralogisms and factious feints. On that basis, the current text intends to use Weiner's book as a starting point to reflect on the conditions of possibility of a rigourous (re)evaluation of ethnographic evidence, as well as those related to the production of ethnographic texts that open themselves to the possibility of further revaluation.
\end{abstract}

Keywords: Annete Weiner - Anthropological Theory - Ethnography - Restudies.

Resumo: Os argumentos que Annette Weiner utiliza em Inalienable Possessions, seu principal trabalho teórico, baseiam-se em um reexame das evidências etnográficas de certas obras, locais e perspectivas clássicas da antropologia à luz de sua própria experiência de campo e da mobilização de conceitos e categorias analíticas contemporâneas. Além dos debates mais conhecidos em torno de alguns famosos "re-estudos" da história da disciplina, as (re) avaliações sistemáticas de argumentos etnográficos como os de Weiner continuam a ser a exceção e não a regra na prática de nosso trabalho, muitas vezes propenso a substituir o reexame das evidências por presenteísmo trivial, paralogismos ingênuos e 
truques facciosos. Com base nisso, o presente texto se propõe a utilizar o livro de Weiner para refletir sobre as condições de possibilidade de uma (re) avaliação rigorosa das evidências etnográficas, bem como aquelas da produção de textos etnográficos que ofereçam a possibilidade de ser reavaliados a posteriori.

Palabras clave: Annete Weiner - Teoria Antropológica - Etnografia - Re-estudos.

[Las traducciones de los abstracts fueron supervisadas por el autor de cada artículo] 